\title{
Conservar Património|
}

ARP | Associação Profissional de Conservadores-Restauradores de Portugal

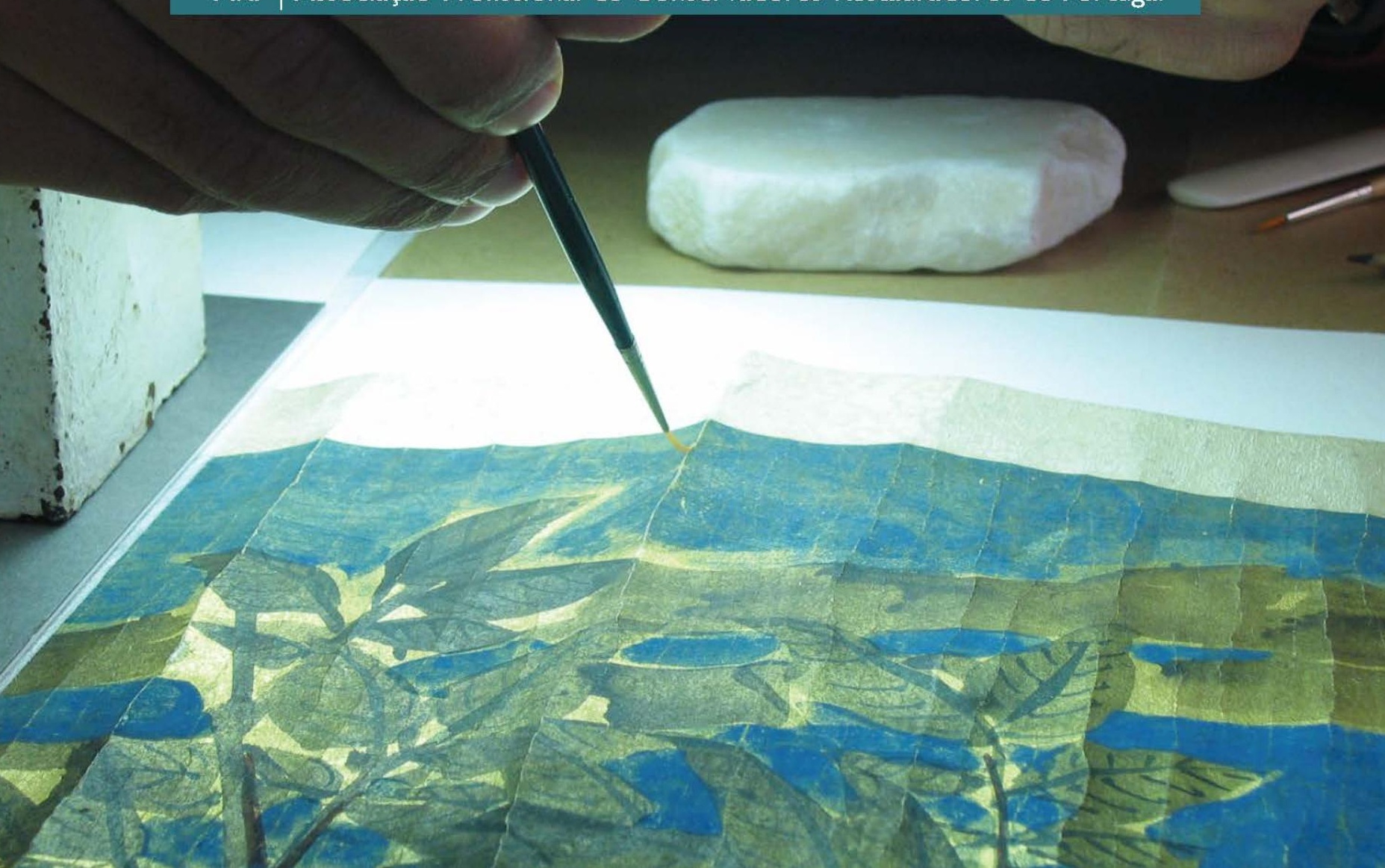





\section{Ficha Técnica_Journal Information}

Edição, propriedade e redacção Publisher and editorial office Associação Profissional de Conservadores-

-Restauradores de Portugal (ARP)

Praça das Amoreiras, n8, R/C, 1250-020 Lisboa

http://revista.arp.org.pt mail@arp.org.pt

Periodicidade Published

Semestral_Biannual

Contribuinte_Tax identification number

503602981

Registo no ICS ICS register number

124638

Depósito Legal_Legal deposit 219614/04

ISSN ISSN

1646-043X

Director Editor

António João Cruz

Sub-Directoras Associate Editors

Francisca Figueira

Maria João Revez

Marketing e Circulação Marketing Rita Horta e Costa, Andreia Ribeiro

Design Gráfico Graphic design Maria da Graça Campelo

Impressão Print

ImpreJornal Sociedade de Impressão, S.A

EN115 ao km80, Quinta Velha, St. Antão do Tojal

Tiragem_Circulation 500 exemplares

Preço geral Public : $8 €$

Preço para instituições Institutional : $40 €$ Preço para sócios da ARP_ Associate Members : $5 €$

As opiniões manifestadas na revista são da exclusiva responsabilidade dos seus autores e não traduzem necessariamente a opinião da ARP ou da Comissão Editorial.

The opinions published in this journal are those of the authors alone and do not necessarily translate the views or opinions of ARP or of its Editorial Board.

Contactos para o envio de colaborações Addresses for sending collaborations António João Cruz ajcruz@ipt.pt

Francisca Figueira Instituto dos Museus e da Conservação Rua das Janelas Verdes, 37 1249-018 Lisboa

FCT Fundação para a Ciência e a Tecnologia MINISTÉRIO DA CIÊNCIA E DO ENSINO SUPERIOR

\section{Artigos e Intervenções |}

Articles and Interventions

3

Acondicionamento de um conjunto de rolos

Chineses da colecção de Camilo Pessanha: uma solução alternativa

Mounting of a group of Chinese scrolls from the Camilo Pessanha collection: an alternative solution

Miguel J. L. Lourenço, João Paulo Dias,

Isabel Zarazúa Astigarraga

11

El desplazamiento de estructuras arqueológicas en España. Trasladar para conservar, conservar para difundir

The displacement of archaeological structures in Spain. Relocate to preserve, preserve to present Víctor Manuel López-Menchero Bendicho

27

Study on the application methods of three adhesives used in the consolidation of contemporary paintings Estudo de métodos de aplicação de três adesivos usados em consolidação de pinturas contemporâneas

Rosario Llamas, Abraham Reina

37

Conservação de um conjunto de pinturas e caligrafias chinesas da colecção de Camilo Pessanha: exemplo de uma abordagem "aquém-oriental"

Conservation of a group of Chinese paintings and calligraphies from the Camilo Pessanha collection: an example of a "less oriental" approach

Miguel J. L. Lourenço, Isabel Zarazúa Astigarraga, João Paulo Dias

59

Estudo e tratamento da obra "Gauguin (o casamento)" de Álvaro Lapa: a problemática da limpeza em pinturas contemporâneas

Study and treatment of the painting "Gauguin (o casamento)", by Álvaro Lapa: cleaning problems found in contemporary paintings

Ana Fryxell, Isabel Ribeiro, Ana Margarida Cardoso, Ana Mesquita e Carmo, António Candeias

69

Normas I Author Guidelines 


\section{Conselho Editorial / Editorial Board}

\section{Ana Calvo}

Professora Associada Convidada, Escola das Artes, Universidade Católica Portuguesa, Porto, Portugal

\section{António CANDEIAS}

Professor Auxiliar, Departamento de Química, Universidade de Évora, Portugal

\section{Mark CLARKE}

Academic Researcher, Universiteit van Amsterdam, Holanda João COROADO

Professor Coordenador, Departamento de Arte, Conservação e Restauro, Instituto Politécnico de Tomar, Portugal

\section{Christian DegrignY}

Conservation Scientist, Haute Ecole de Conservation-Restauration Arc, La Chaux-de-Fonds, Suiça

\section{Ester FERREIRA}

Head of the Analytical Research Laboratory of the Art Technology, Schweizerisches Institut für Kunstwissenschaft, Zürich, Suiça

\section{M. ${ }^{a}$ José GONZÁLEZ LÓPEZ}

Profesora Titular, Departamento de Pintura, Facultad de Bellas Artes, Universidad de Sevilla, Espanha

\section{René LARSON}

Rector of School of Conservation, The Royal Danish Academy of Fine Arts, Copenhagen; Presidente da rede ENCoRE, European Network for Conservation-Restoration Education, Dinamarca

\section{Ana Martins}

Associate Research Scientist, Conservation Department, Museum of Modern Art, New York, USA

Edson MotтA

Professor, Universidade Federal do Rio de Janeiro, Brasil

\section{Salvador MUÑoz ViñAS}

Professor, Universidad Politécnica de Valencia, Espanha

\section{Mário Mendonça de Oliveira}

Professor, Universidade Federal da Bahia, Brasil

\section{Vítor SERRÃo}

Professor Catedrático, Instituto de História da Arte, Faculdade de Letras da Universidade de Lisboa, Portugal

\section{Rosário VEIGA}

Investigadora Principal com Habilitação para Coordenação Científica, Laboratório Nacional de Engenharia Civil, Lisboa, Portugal
Material adicional dos artigos está livremente disponível no site da revista

Additional material is freely available at the journal website

http://revista.arp.org.pt/

Normas de colaboração e instruções para os autores

http://revista.arp.org.pt/pt/normas.html

Author guidelines

http://revista.arp.org.pt/en/normas.html

Fotografia da capa

Cover photography

Miguel J. L. Lourenço, Isabel Zarazúa Astigarraga, João Paulo Dias

\section{A revista está indexada em}

The journal is indexed in

>AATA - Art and Archaeology Technical Abstracts,

Getty Conservation Institute

$>$ Chemical Abstracts, American Chemical Society

>BCIN - The Bibliographic Database of the

Conservation Information Network

A revista está referenciada em

The journal is referenced in

> Latindex - Sistema Regional de Información en

Línea para Revistas Científicas de América Latina,

el Caribe, España y Portugal 